\title{
I-optimal design of mixture experiments
}

\author{
Peter Goos \\ KU Leuven \\ Universiteit Antwerpen \\ Bradley Jones \\ SAS Institute Inc. \\ Universiteit Antwerpen \\ Utami Syafitri \\ Universiteit Antwerpen
}

\begin{abstract}
In mixture experiments, the factors under study are proportions of the ingredients of a mixture. The special nature of the factors necessitates specific types of regression models, and specific types of experimental designs. Although mixture experiments usually are intended to predict the response(s) for all possible formulations of the mixture and to identify optimal proportions for each of the ingredients, little research has been done concerning their I-optimal design. This is surprising given that I-optimal designs minimize the average variance of prediction and, therefore, seem more appropriate for mixture experiments than the commonly used D-optimal designs, which focus on a precise model estimation rather than precise predictions. In this paper, we provide the first detailed overview of the literature on the I-optimal design of mixture experiments and identify several contradictions. For the second-order and the special cubic model, we present I-optimal continuous designs and contrast them with the published results. We also study exact
\end{abstract}


I-optimal designs, and compare them in detail to continuous I-optimal designs and to D-optimal designs. One striking result of our work is that the performance of D-optimal designs in terms of the I-optimality criterion very strongly depends on which of the D-optimal designs is considered. Supplemental materials for this article are available online.

Keywords: D-optimality, IV-optimality, moments matrix, Q-optimality, Scheffé model, simplex-lattice designs, simplex-centroid designs, V-optimality.

\section{Introduction}

In recent years, prediction-based optimality criteria have gained substantial popularity for generating response surface designs. The best-known prediction-based optimality criteria are the G-optimality criterion, which seeks designs that minimize the maximum prediction variance over the experimental region, and the I-optimality criterion, which seeks designs that minimize the average prediction variance over the experimental region. Within the optimal experimental design community, the I-optimality criterion is often called the V-optimality criterion (see, for instance, Atkinson, Donev and Tobias (2007)), but the names IV- or Q-optimality have been used as well (Borkowski, 2003; Letsinger, Myers and Lentner, 1996). In this paper, we refer to designs that minimize the average prediction variance as I-optimal designs. Concerning the I- and G-optimality criteria, Montgomery (2009) writes that "they would be most likely used for second-order models, as second-order models are often used for optimization, and good prediction properties are essential for optimization." The increasing use of variance dispersion graphs and fraction of design space plots (Giovannitti-Jensen and Myers, 1989; Zahran and Anderson-Cook, 2003; Goldfarb, Anderson-Cook, Borror and Montgomery, 2004) has contributed to the increasing focus on prediction variances when selecting experimental designs. 
The generation of I-optimal completely randomized response surface designs is discussed in Haines (1987), Meyer and Nachtsheim (1988, 1995), Hardin and Sloane (1993) and Borkowski (2003). The generation of G-optimal completely randomized designs is treated in Borkowski (2003) and Rodríguez et al. (2010). The latter authors point out that, to minimize the maximum variance of prediction, it is often necessary to accept larger prediction variances over most of the region of interest, and suggest using the I-optimality criterion rather than the G-optimality criterion. In this article, we therefore focus on the I-optimality criterion.

Despite the increasing use of the I-optimality criterion for response surface designs, little is known concerning I-optimal mixture designs. This is surprising for two reasons:

1. In his seminal paper on mixture experiments, Scheffé (1958) already suggests using the variance of the predicted response as a starting point to design mixture experiments. In follow-up work, Lambrakis (1968a,b) formally defines an I-optimality criterion for two special types of mixture designs, which are not commonly used, but he did not succeed in constructing I-optimal designs due to the fact that the required analytical expressions were intractable and the available computing power was limited.

2. Mixture designs are special cases of response surface designs, and prediction and optimization are the main goals of most mixture experiments. The two textbooks on the design of mixture experiments (Cornell, 2002; Smith, 2005) mention the Ioptimality criterion briefly, but they do not discuss I-optimal mixture designs in any detail. The reference books by Atkinson et al. (2007) and Goos and Jones (2011) briefly discuss the D-optimality of some commonly used mixture designs, but pay no attention to I-optimal mixture designs. 
The only published results concerning I-optimal mixture designs for Scheffé models can be found in Lambrakis (1968b), Laake (1975), Liu and Neudecker (1995) and Sinha, Mandal, Pal and Das (2014). I-optimal designs for the models of Becker (1968) are discussed in Liu and Neudecker (1997). In this paper, we focus on the most commonly used Scheffé models. As did Scheffé $(1958,1963)$, we also concentrate on simplex-shaped experimental regions.

Throughout this paper, we distinguish between continuous optimal designs and exact optimal designs. Exact designs are intended for experiments with a finite number of observations or runs, $n$, and involve $n$ design points, which are not necessarily distinct. Continuous designs involve a set of distinct design points and a weight for each of these points. The weight of each design point in a continuous design indicates the proportion of experimental tests to be performed at that point. Continuous designs have received much attention in the literature on the optimal design of experiments, because their optimality can be established using the general equivalence theorem (Kiefer and Wolfowitz; 1960) and because continuous optimal designs provide good guidance for the optimal selection of design points when the number of runs available is large. For the D-optimal design of mixture experiments, the continuous D-optimal designs provide perfect guidance even when the number of runs available is small. In this paper, after identifying the continuous I-optimal designs, we investigate whether they also provide good guidance when setting up small mixture experiments.

We introduce the Scheffé models in Section 2. Next, in Section 3, we define the D- and I-optimality criteria for continuous and for exact designs. We summarize the existing theoretical results concerning D- and I-optimality in Section 4. In Section 5, we present continuous I-optimal designs for the second-order and special cubic models and show that 
they outperform the I-optimal arrangements presented by Laake (1975). In Section 6, we discuss exact I-optimal designs and contrast them with continuous I-optimal designs. Also, we provide a detailed comparison of exact I-optimal mixture designs and exact Doptimal mixture designs in terms of the variance of prediction. In the paper's final section, we summarize the key results and discuss the usefulness of the SAS procedure OPTEx for generating I-optimal designs.

\section{Statistical models and analysis}

In models for data from mixture experiments involving $q$ ingredients, the explanatory variables are the $q$ ingredient proportions $x_{1}, x_{2}, \ldots, x_{q}$. A key feature of these explanatory variables is that they sum to one: $\sum_{i=1}^{q} x_{i}=\mathbf{x}^{\prime} \mathbf{1}_{q}=1$, where $\mathbf{x}^{\prime}=\left(x_{1}, x_{2}, \ldots, x_{q}\right)$ and $\mathbf{1}_{q}$ is a $q$-dimensional vector of ones. This constraint, which is called the mixture constraint, defines a simplex-shaped experimental region and has a substantial impact on the models that can be fitted. The first major consequence of the mixture constraint is that a regression model involving linear terms in the ingredient proportions cannot contain an intercept. Otherwise, the model's parameters cannot be estimated uniquely. A second major consequence is that including cross-products of proportions together with squares of proportions should be avoided too, because this also results in the model parameters not being estimable uniquely. To see this, note that

$$
x_{i}^{2}=x_{i}\left(1-\sum_{\substack{j=1 \\ j \neq i}}^{q} x_{j}\right)=x_{i}-\sum_{\substack{j=1 \\ j \neq i}}^{q} x_{i} x_{j},
$$

for every proportion $x_{i}$. As a result, the square of a proportion is a linear combination of that proportion and its cross-products with each one of the other $q-1$ proportions. 
These considerations led Scheffé (1958) to propose the Scheffé mixture models. The firstorder Scheffé model is given by

$$
E(Y)=\sum_{i=1}^{q} \beta_{i} x_{i}
$$

whereas the second-order Scheffé model is given by

$$
E(Y)=\sum_{i=1}^{q} \beta_{i} x_{i}+\sum_{i=1}^{q-1} \sum_{j=i+1}^{q} \beta_{i j} x_{i} x_{j}
$$

The special cubic model can be written as

$$
E(Y)=\sum_{i=1}^{q} \beta_{i} x_{i}+\sum_{i=1}^{q-1} \sum_{j=i+1}^{q} \beta_{i j} x_{i} x_{j}+\sum_{i=1}^{q-2} \sum_{j=i+1}^{q-1} \sum_{k=j+1}^{q} \beta_{i j k} x_{i} x_{j} x_{k}
$$

and the full cubic model is

$$
\begin{aligned}
E(Y)= & \sum_{i=1}^{q} \beta_{i} x_{i}+\sum_{i=1}^{q-1} \sum_{j=i+1}^{q} \beta_{i j} x_{i} x_{j}+\sum_{i=1}^{q-1} \sum_{j=i+1}^{q} \gamma_{i j} x_{i} x_{j}\left(x_{i}-x_{j}\right) \\
& +\sum_{i=1}^{q-2} \sum_{j=i+1}^{q-1} \sum_{k=j+1}^{q} \beta_{i j k} x_{i} x_{j} x_{k} .
\end{aligned}
$$

Another model that has received attention in the literature (Scheffé, 1963) is the qth degree model. This model adds specific higher-order terms to the special cubic model, but it is not commonly used (see, for example, Cornell (2002) and Smith (2005)). Except for a few specific results, we therefore do not discuss I-optimal designs for the $q$ th degree model in detail in this paper. Instead, we summarize our results for that model in an online supplemental file. 


\section{Criteria for selecting designs}

In this section, we define the prediction-oriented I-optimality criterion, which is central to this paper. We pay special attention to the computation of the I-optimality criterion because popular implementations of the criterion are based on a grid of points covering the experimental region, and these implementations can be improved upon substantially by taking an analytical approach. We also define the estimation-oriented D-optimality criterion because we use D-optimal designs as benchmarks later in this paper.

\subsection{D-optimality criterion}

The most commonly used optimality criterion to select designs is the estimation-oriented D-optimality criterion which seeks designs that maximize the determinant of the information matrix, and, in doing so, minimize the volume of the confidence ellipsoid about the unknown model parameters. For exact designs, the information matrix is computed as $\mathbf{M}=\mathbf{X}^{\prime} \mathbf{X}$, where $\mathbf{X}$ is the $n \times p$ model matrix, with $n$ the number of runs and $p$ the number of terms in the model. For continuous designs involving $d$ distinct design points, the information matrix is given by $\mathbf{M}=\mathbf{X}_{d}^{\prime} \mathbf{W} \mathbf{X}_{d}$, where $\mathbf{X}_{d}$ is the $d \times p$ model matrix corresponding to the $d$ design points, $\mathbf{W}=\operatorname{diag}\left(w_{1}, w_{2}, \ldots, w_{d}\right)$ is a diagonal matrix whose diagonal elements are the weights of the $d$ design points, and $\sum_{i=1}^{d} w_{i}=1$.

We use the D-efficiency to compare the quality of two (continuous or exact) designs with information matrices $\mathbf{M}_{1}$ and $\mathbf{M}_{2}$. The D-efficiency of a design with information matrix $\mathbf{M}_{1}$ relative to a design with information matrix $\mathbf{M}_{2}$ is defined as $\left(\left|\mathbf{M}_{1}\right| /\left|\mathbf{M}_{2}\right|\right)^{1 / p}$. $\mathbf{A}$ D-efficiency larger than one indicates that Design 1 is better than Design 2 in terms of the D-optimality criterion. 
Due to the general equivalence theorem (Kiefer and Wolfowitz, 1960), continuous Doptimal designs are also G-optimal, which means they minimize the maximum prediction variance $\mathbf{f}^{\prime}(\mathbf{x}) \mathbf{M}^{-1} \mathbf{f}(\mathbf{x})$, where $\mathbf{x}$ is a combination of mixture proportions and $\mathbf{f}(\mathbf{x})$ represents its model expansion, over the experimental region $\chi$. This equivalence is, however, not generally valid for exact designs.

\section{$3.2 \quad$ I-optimality criterion}

An I-optimal design minimizes the average prediction variance

$$
\text { Average variance }=\frac{\int_{\chi} \mathbf{f}^{\prime}(\mathbf{x}) \mathbf{M}^{-1} \mathbf{f}(\mathbf{x}) d \mathbf{x}}{\int_{\chi} d \mathbf{x}}
$$

over the experimental region $\chi$. To calculate this average variance, we exploit the fact that, when calculating the trace of a matrix product, we can cyclically permute the matrices. Therefore, we can rewrite the formula for the average prediction variance as

$$
\text { Average variance }=\frac{1}{\int_{\chi} d \mathbf{x}} \cdot \operatorname{tr}\left[\mathbf{M}^{-1} \mathbf{B}\right]
$$

where

$$
\mathbf{B}=\int_{\chi} \mathbf{f}(\mathbf{x}) \mathbf{f}^{\prime}(\mathbf{x}) d \mathbf{x}
$$

This matrix is called the moments matrix because its elements are proportional to moments of a uniform distribution on the experimental region $\chi$. Assuming the experimental region is the full $(q-1)$-dimensional simplex, the elements of $\mathbf{B}$ can be obtained using the formula

$$
\int_{S_{q-1}} x_{1}^{p_{1}} x_{2}^{p_{2}} \ldots x_{q}^{p_{q}} d x_{1} d x_{2} \ldots d x_{q}=\frac{\prod_{i=1}^{q} \Gamma\left(p_{i}+1\right)}{\Gamma\left(q+\sum_{i=1}^{q} p_{i}\right)}
$$

which is given, for instance, in De Groot (1970, page 63), but which also appeared, albeit in a different notation, in Lambrakis (1968a,b). As an example, for a special cubic model 
involving three ingredients, the moments matrix equals

$$
\mathbf{B}=\left[\begin{array}{ccc}
\frac{1}{24}\left(\mathbf{I}_{3}+\mathbf{1}_{3} \mathbf{1}_{3}^{\prime}\right) & \frac{1}{60} \mathbf{1}_{3} \mathbf{1}_{3}^{\prime}-\frac{1}{120} \mathbf{J}_{3} & \frac{1}{360} \mathbf{1}_{3} \\
\frac{1}{60} \mathbf{1}_{3} \mathbf{1}_{3}^{\prime}-\frac{1}{120} \mathbf{J}_{3} & \frac{1}{360}\left(\mathbf{I}_{3}+\mathbf{1}_{3} \mathbf{1}_{3}^{\prime}\right) & \frac{1}{1260} \mathbf{1}_{3} \\
\frac{1}{360} \mathbf{1}_{3}^{\prime} & \frac{1}{1260} \mathbf{1}_{3}^{\prime} & \frac{1}{5040}
\end{array}\right]
$$

where $\mathbf{I}_{3}$ is the three-dimensional identity matrix, $\mathbf{J}_{3}$ is the three-dimensional anti-diagonal identity matrix, and $\mathbf{1}_{3}$ is a three-dimensional column vector of ones. In Equation (9), the expressions on the diagonal correspond to the three main effects, the three second-order terms and the cubic term, respectively. When the experimental region is the $(q-1)$ dimensional simplex, then its volume equals

$$
\int_{\chi} d \mathbf{x}=\int_{S_{q-1}} d \mathbf{x}=\frac{1}{\Gamma(q)}
$$

If $P_{1}$ is the average variance of prediction of one design and $P_{2}$ is the average variance of prediction of a second design, then the I-efficiency of the former design compared to the latter is computed as $P_{2} / P_{1}$. An I-efficiency larger than one indicates that Design 1 is better than Design 2 in terms of the average prediction variance.

\section{Review of D- and I-optimal mixture designs}

In this section, we provide an overview of the published results on D- and I-optimal mixture designs. We refer readers who are interested in mixture designs that are optimal with respect to other criteria to Chan (1995) and Sinha et al. (2014). It is striking that both continuous and exact D-optimal designs are known, but that, for the I-optimality criterion, only continuous designs have been studied in detail. Key concepts in the overview in this section are two types of mixture designs called simplex-lattice designs and simplex- 
centroid designs. The points of these designs play a crucial role in continuous optimal designs, and, for this reason, they are often used as candidate sets for point-exchange algorithms for constructing exact designs.

\subsection{Simplex-lattice and simplex-centroid designs}

\subsubsection{Simplex-lattice designs}

A $\{q, m\}$ simplex-lattice design for $q$ ingredients involves all possible mixture formulations, the $q$ individual ingredient proportions of which belong to the set $\left\{0, \frac{1}{m}, \frac{2}{m}, \ldots, 1\right\}$. In total, there are

$$
\left(\begin{array}{c}
m+q-1 \\
m
\end{array}\right)
$$

points in a $\{q, m\}$ simplex-lattice design. For instance, a $\{3,1\}$ simplex-lattice design involves three design points, $(1,0,0),(0,1,0)$ and $(0,0,1)$. We call these points pure components. A $\{3,2\}$ simplex-lattice design involves six design points, the pure components as well as the points $\left(\frac{1}{2}, \frac{1}{2}, 0\right),\left(\frac{1}{2}, 0, \frac{1}{2}\right)$ and $\left(0, \frac{1}{2}, \frac{1}{2}\right)$. We call the latter points, involving $50 \%$ of one ingredient and $50 \%$ of another, binary mixtures in this paper. In general, there are $q$ pure components and $\left(\begin{array}{l}q \\ 2\end{array}\right)=n(n-1) / 2$ binary mixtures.

\subsubsection{Simplex-centroid designs}

The full simplex-centroid design involves $2^{q}-1$ design points: the $q$ pure components, the $\left(\begin{array}{l}q \\ 2\end{array}\right)$ binary mixtures, the $\left(\begin{array}{l}q \\ 3\end{array}\right)$ permutations of the ternary mixture $\left(\frac{1}{3}, \frac{1}{3}, \frac{1}{3}, 0, \ldots, 0\right)$, $\ldots$, the $q$ permutations of the mixture $\left(\frac{1}{q-1}, \frac{1}{q-1}, \ldots, \frac{1}{q-1}, 0\right)$, and, finally, the mixture $\left(\frac{1}{q}, \frac{1}{q}, \ldots, \frac{1}{q}\right)$ involving an equal proportion of all $q$ components.

For every number of ingredients $q$, there is only one simplex centroid design, but there is a family of simplex-lattice designs. The simplex-centroid design involves the overall 
centroid, and the centroids of all lower dimensional simplices. An important fraction of the simplex-centroid design consists of the pure components, the binary mixtures and the ternary mixtures. That fraction can be used to estimate the special cubic model. We refer to this fraction as the $\{q, 3\}$ simplex-centroid design. A larger fraction of the simplex-centroid design also involves the quaternary and quinary mixtures. We refer to that fraction as the $\{q, 5\}$ simplex-centroid design.

\subsection{Existing results on D-optimality}

\subsubsection{Continuous D-optimal designs}

Continuous D-optimal designs for the models (1)-(4) are known in case the experimental region is the $(q-1)$-dimensional simplex. The D-optimal designs have two key features. First, they are minimum support designs, meaning that the number of distinct design points, $d$, equals the number of model parameters, $p$. Second, the weight of each design point in the D-optimal designs is $1 / p$.

The D-optimality of the $\{q, 1\}$ and $\{q, 2\}$ simplex-lattice designs for models (1) and (2), respectively, was established by Kiefer (1961). Uranisi (1964) showed that the $\{q, 3\}$ simplex-centroid designs are D-optimal for special cubic models. For the full cubic model, the D-optimal designs involve

- the $q$ pure components,

- the $2\left(\begin{array}{l}q \\ 2\end{array}\right)$ mixtures involving $27.64 \%$ of one ingredient and $72.36 \%$ of another ingredient (the exact proportions are given by $(1 \pm 1 / \sqrt{5}) / 2$ ), and

- the $\left(\begin{array}{l}q \\ 3\end{array}\right)$ ternary mixtures.

So, D-optimal designs for full cubic models can be constructed by replacing the proportions 
$1 / 3$ and $2 / 3$ of the $\{q, 3\}$ simplex-lattice designs by 0.2764 and 0.7236 , respectively. A general proof for the D-optimality of these designs for the full cubic model is given by Mikaeili (1993). For $q$ th degree models, Atwood (1969) showed that the D-optimal points are those of the full simplex-centroid designs. Each of the points should have weight $\left(2^{q}-1\right)^{-1}$.

\subsubsection{Exact D-optimal designs}

Exact D-optimal designs involve the same $d=p$ design points as D-optimal continuous designs. If the budgeted number of runs, $n$, in a mixture experiment is an integer multiple of the number of points of the continuous D-optimal design, then $n / p=n / d$ runs have to be performed at each of these points. If $n$ is not an integer multiple of $d=p$, then the $d=p$ design points should be as equireplicated as possible. Which points are replicated most frequently is unimportant, provided the interest is in the D-optimality criterion only. In Section 6, we demonstrate that some D-optimal designs perform excellently in terms of the I-optimality criterion, while others perform poorly.

\subsection{Existing results on I-optimality}

A limited number of theoretical results have been published concerning the I-optimal design of mixture experiments. All of the results involve continuous designs. In this section, we provide a tabular overview and point out a few conflicting results.

\subsubsection{First-order model}

Theorem 12.1.1 of Sinha et al. (2014), who interchangeably use the terms V-optimal, Ioptimal and all-variance design to refer to I-optimal designs, states that the continuous I-optimal design for a first-order model in $q$ ingredients has a weight of $1 / q$ at each point of the $\{q, 1\}$ simplex-lattice design. The optimal design points thus are the $q$ pure 
Table 1: I-optimal weights for a second-order model according to Laake (1975), assuming the $\{q, 2\}$ simplex-lattice design points are used.

\begin{tabular}{|c|ccc|ccc|ccc|ccc|}
\hline \multirow{2}{*}{$i$} & \multicolumn{4}{|c|}{$q=3$} & \multicolumn{4}{|c|}{$q=4$} & \multicolumn{3}{c|}{$q=5$} & \multicolumn{3}{c|}{$q=6$} \\
\cline { 2 - 12 } & $n_{i}$ & $w_{i}$ & $n_{i} w_{i}$ & $n_{i}$ & $w_{i}$ & $n_{i} w_{i}$ & $n_{i}$ & $w_{i}$ & $n_{i} w_{i}$ & $n_{i}$ & $w_{i}$ & $n_{i} w_{i}$ \\
\hline 1 & 3 & 0.1007 & 0.3022 & 4 & 0.0560 & 0.2240 & 5 & 0.0400 & 0.2000 & 6 & 0.0328 & 0.1968 \\
2 & 3 & 0.2326 & 0.6978 & 6 & 0.1293 & 0.7760 & 10 & 0.0800 & 0.8000 & 15 & 0.0536 & 0.8032 \\
\hline$d$ & \multicolumn{2}{|r|}{6} & \multicolumn{4}{|c|}{10} & & \multicolumn{3}{|c|}{15} & & \multicolumn{3}{|c|}{21} \\
\hline
\end{tabular}

components, and each of these should be used equally often.

\subsubsection{Second-order model}

Laake (1975) analytically derived the I-optimal weights for the designs points in case $q \geq 3$ and a second-order model is estimated, assuming that the design points are those of the $\{q, 2\}$ simplex-lattice design. Thus, Laake's weights are I-optimal contingent on the $\{q, 2\}$ simplex-lattice design being used. The analytical expressions he obtained for the weights are given in the online supplemental file. Numerical values for the weights for values of $q$ from 3 to 6 are given in Table 1 . The weight $w_{1}$ indicates the proportion of runs to be conducted with each pure component, whereas the weight $w_{2}$ indicates the proportion of runs to be done with each binary mixture. The table also lists the number of pure components $\left(n_{1}\right)$ and the number of binary mixtures $\left(n_{2}\right)$, as well as the proportion of experimental runs involving pure components $\left(n_{1} w_{1}\right)$ and binary mixtures $\left(n_{2} w_{2}\right)$. The table's last line contains the number of distinct design points, $d=n_{1}+n_{2}$.

Unlike in the continuous D-optimal designs for the second-order model, each pure component in Laake's design has a weight of less than $1 / d$, while each binary mixture has a weight of more than $1 / d$. Roughly $20 \%$ of the experimental effort involves pure components, whereas $80 \%$ involves binary mixtures when $q \geq 4$.

Laake (1975) did not mention the case of two ingredients $(q=2)$ when discussing the 
Table 2: I-optimal weights for a special cubic model according to Laake (1975), assuming the $\{q, 3\}$ simplex-centroid design points are used.

\begin{tabular}{|c|c|c|c|c|c|c|c|c|c|c|c|c|}
\hline \multirow{2}{*}{$\imath$} & \multicolumn{3}{|c|}{$q=3$} & \multicolumn{3}{|c|}{$q=4$} & \multicolumn{3}{|c|}{$q=5$} & \multicolumn{3}{|c|}{$q=6$} \\
\hline & $n_{i}$ & $w_{i}$ & $n_{i} w_{i}$ & $n_{i}$ & $w_{i}$ & $n_{i} w_{i}$ & $n_{i}$ & $w_{i}$ & $n_{i} w_{i}$ & $n_{i}$ & $w_{i}$ & $n_{i} w_{i}$ \\
\hline 1 & 3 & 0.0925 & 0.2776 & 4 & 0.0417 & 0.1667 & 5 & 0.0212 & 0.1060 & 6 & 0.0121 & 0.0726 \\
\hline 2 & 3 & 0.1483 & 0.4448 & 6 & 0.0556 & 0.3333 & 10 & 0.0275 & 0.2751 & 15 & 0.0177 & 0.2653 \\
\hline 3 & 1 & 0.2776 & 0.2776 & 4 & 0.1250 & 0.5000 & 10 & 0.0619 & 0.6189 & 20 & 0.0331 & 0.6621 \\
\hline$d$ & & 7 & & & 14 & & & 25 & & & 41 & \\
\hline
\end{tabular}

second-order model. The second-order model for $q=2$ is, however, a special case of the $q$ th degree model, for which Liu and Neudecker (1995) analytically derive continuous Ioptimal designs. Their analytical expressions yield $w_{1}=0.3$ and $w_{2}=0.4$ as the weights for the two pure components and the binary mixture.

Prior to Laake (1975), Lambrakis (1968a) considered the I-optimal assignment of weights to the points of a specific, unusual design. In terms of the I-optimality criterion, the designs advocated by Laake (1975) are far superior to those proposed by Lambrakis (1968a).

\subsubsection{Special cubic model}

Laake (1975) also presents I-optimal weights for the special cubic model, assuming the design points are those of the $\{q, 3\}$ simplex-centroid design. This time, Laake's weights are I-optimal contingent on the $\{q, 3\}$ simplex-centroid design being used. The analytical expressions he obtained for the weights are given in the online supplemental file. Numerical values for the weights for $q$ ranging from 3 to 6 are given in Table 2 , where $n_{1}, n_{2}, w_{1}$ and $w_{2}$ have the same meaning as in the previous table and $w_{3}$ is the proportion of runs with each ternary mixture. Table 2's final line gives the number of distinct design points considered, $d=n_{1}+n_{2}+n_{3}$.

The key difference between the continuous D-optimal designs for the special cubic model 
and the designs suggested by Laake (1975) is that the latter attach substantially more weight to the ternary mixtures. This can be seen from the fact that $n_{3} w_{3}$, the proportion of runs using ternary mixtures in Table 2 , is substantially larger than $n_{3} / d$, which is the proportion of runs using ternary mixtures in the D-optimal continuous designs.

As the special cubic model for $q=3$ is a special case of the $q$ th degree model, we can also consult Liu and Neudecker (1995) for that case. Their analytical expressions yield $w_{1}=0.1383, w_{2}=0.1345$ and $w_{3}=0.1816$ as the weights for each pure component, for each binary mixture and for the ternary mixture, respectively. Remarkably, these weights differ from those of Laake (1975), even though the same set of design points is considered. Calculating the average prediction variances for the two designs leads to a value of 4.1315 for Liu and Neudecker's design and 3.7543 for Laake's design, so that Liu and Neudecker's design is inferior and has an I-efficiency of $90.87 \%$ only, relative to Laake's design.

\subsubsection{Full cubic model}

The final general results presented by Laake (1975) involve the I-optimal weights for the full cubic model, assuming the design points are those of the $\{q, 3\}$ simplex-lattice design. The analytical expressions he obtained for the weights $w_{1}$ of the pure components, $w_{2}$ of the mixtures involving $1 / 3$ of one ingredient and $2 / 3$ of another ingredient, and $w_{3}$ of the ternary mixtures involving $1 / 3$ of three different ingredients are given in the online supplemental file. Numerical values for the weights are given in Table 3.

\section{Continuous I-optimal designs}

In this section, we present continuous I-optimal designs and contrast them with the results reported in the literature and reviewed in Section 4. To verify the optimality of our 
Table 3: I-optimal weights for a full cubic model according to Laake (1975), assuming the $\{q, 3\}$ simplex-lattice design points are used.

\begin{tabular}{|c|c|c|c|c|c|c|c|c|c|c|c|c|}
\hline \multirow{2}{*}{$i$} & \multicolumn{3}{|c|}{$q=3$} & \multicolumn{3}{|c|}{$q=4$} & \multicolumn{3}{|c|}{$q=5$} & \multicolumn{3}{|c|}{$q=6$} \\
\hline & $n_{i}$ & $w_{i}$ & $n_{i} w_{i}$ & $n_{i}$ & $w_{i}$ & $n_{i} w_{i}$ & $n_{i}$ & $w_{i}$ & $n_{i} w_{i}$ & $n_{i}$ & $w_{i}$ & $n_{i} w_{i}$ \\
\hline 1 & 3 & 0.0416 & 0.1247 & 4 & 0.0179 & 0.0715 & 5 & 0.0117 & 0.0585 & 6 & 0.0093 & 0.0556 \\
\hline 2 & 6 & 0.1108 & 0.6650 & 12 & 0.0464 & 0.5571 & 20 & 0.0235 & 0.4708 & 30 & 0.0139 & 0.4170 \\
\hline 3 & 1 & 0.2103 & 0.2103 & 4 & 0.0929 & 0.3714 & 10 & 0.0471 & 0.4708 & 20 & 0.0264 & 0.5274 \\
\hline$d$ & & 10 & & & 20 & & & 35 & & & 56 & \\
\hline
\end{tabular}

designs, we used the general equivalence theorem. To find the designs, we used the multiplicative algorithm.

\subsection{Methodology}

\subsubsection{General equivalence theorem for I-optimality}

As explained in, for example, Section 9.2 of Atkinson et al. (2007), the general equivalence theorem provides a methodology to check the optimality of a given continuous design, for any convex and differentiable design optimality criterion. The best-known special case of the general equivalence theorem states that the maximum prediction variance of Doptimal continuous designs equals the number of model parameters. A corollary of that special case is that continuous D-optimal and continuous G-optimal designs are equivalent.

The equivalence theorem can, however, also be applied to the I-optimality criterion. Atkinson et al. (2007) explain that the I-optimality criterion is a special case of the Loptimality criterion (this criterion is also called the linear optimality criterion and seeks designs that minimize the average variance of a set of linear combinations of model parameter estimates). Therefore, a continuous design with information matrix $\mathbf{M}$ is I-optimal if and only if

$$
\mathbf{f}^{\prime}(\mathbf{x}) \mathbf{M}^{-1} \mathbf{B} \mathbf{M}^{-1} \mathbf{f}(\mathbf{x}) \leq \operatorname{tr}\left(\mathbf{M}^{-1} \mathbf{B}\right)
$$


for each point $\mathbf{x}$ in the experimental region $\chi$. The equality in (10) holds only for the points of the design.

We have used the equivalence theorem to verify the I-optimality of the designs for firstorder, second-order and special cubic models we present in this section and the designs for the $q$ th degree model in the online supplemental file. Except for the first-order model, we took a numerical approach, in which we randomly generated 10000 points from a uniform distribution over the experimental region $\chi$. To randomly generate a point from a uniform distribution on a $(q-1)$-dimensional simplex, $q$ observations can be drawn from the standard exponential distribution and the resulting observations can be rescaled so they sum to one. For each of the 10000 points we generated in this fashion, we computed the left-hand side of the inequality in (10) and noted that it is smaller than the right-hand side. For all the design points, we also computed the left-hand side of (10) and noted that it is equal to the inequality's right-hand side.

\subsubsection{Multiplicative algorithm}

To find continuous I-optimal designs, we adapted the multiplicative algorithm to deal with the I-optimality criterion. That algorithm was introduced in the literature by Titterington $(1976,1978)$ and Silvey (1980) as an alternative to the algorithm of Wynn (1972). The strength of our version of the algorithm is that each iteration is based on the fact that, for the I-optimal design, the equality in (10) holds for each design point. Because the algorithm exploits the general equivalence theorem, it converges to the optimal design within seconds for the problems we studied. To the best of our knowledge, we are the first authors to apply the multiplicative algorithm to find I-optimal designs. Our version of the algorithm is outlined in the appendix to this paper. 


\section{$5.2 \quad$ First-order model}

The I-optimal continuous designs for the first-order model involve the points of the $\{q, 1\}$ simplex-lattice designs with equal weight, $1 / q$, for each of them. This can be verified using the general equivalence theorem. First, note that $\mathbf{X}=\mathbf{I}_{q}, \mathbf{W}=q^{-1} \mathbf{I}_{q}$ and $\mathbf{M}=q^{-1} \mathbf{I}_{q}$ for a $\{q, 1\}$ simplex-lattice design with weight $1 / q$ for each of the $q$ design points, and that the moments matrix $\mathbf{B}$ equals $c\left(\mathbf{I}_{q}+\mathbf{1}_{q} \mathbf{1}_{q}^{\prime}\right)$, where $c$ is a constant for any given $q$ value. Hence, the right-hand side of (10) equals $c q \operatorname{tr}\left(\mathbf{I}_{q}+\mathbf{1}_{q} \mathbf{1}_{q}^{\prime}\right)=2 c q^{2}$. Since, for the first-order model, $\mathbf{f}(\mathbf{x})=\mathbf{x}$, and since $\mathbf{x}^{\prime} \mathbf{1}_{q}=\mathbf{1}_{q}^{\prime} \mathbf{x}=1$, the left-hand side of (10) simplifies to

$$
\mathbf{f}^{\prime}(\mathbf{x}) \mathbf{M}^{-1} \mathbf{B} \mathbf{M}^{-1} \mathbf{f}(\mathbf{x})=c q^{2} \mathbf{x}^{\prime}\left(\mathbf{I}_{q}+\mathbf{1}_{q} \mathbf{1}_{q}^{\prime}\right) \mathbf{x}=c q^{2}\left(\mathbf{x}^{\prime} \mathbf{x}+1\right)
$$

Since the elements of $\mathbf{x}$ all lie between zero and one, and sum to one, $\mathbf{x}^{\prime} \mathbf{x}$ is always smaller than or equal to one. The equality only holds if $\mathbf{x}$ represents a pure component (in which case one element of $\mathbf{x}$ is one and all others are zero, $\mathbf{x}^{\prime} \mathbf{x}=1$ and $\left.c q^{2}\left(\mathbf{x}^{\prime} \mathbf{x}+1\right)=2 c q^{2}\right)$. For any other point in the simplex, $\mathbf{x}^{\prime} \mathbf{x}<1$ and $c q^{2}\left(\mathbf{x}^{\prime} \mathbf{x}+1\right)<2 c q^{2}$. The $\{q, 1\}$ simplex-lattice designs with weight $1 / q$ for each point therefore satisfy the general equivalence theorem, so that they are I-optimal.

\subsection{Second-order model}

While the continuous D-optimal designs' points for second-order models equal those of the $\{q, 2\}$ simplex-lattice designs, the continuous I-optimal designs'points for $q \geq 3$ are those of the $\{q, 3\}$ simplex-centroid designs. The I-optimal weights for the pure components, the binary mixtures and the ternary mixtures, rounded to four decimal places, are shown in Table 4. The I-efficiencies of the designs proposed by Laake (1975) relative to the I-optimal designs we obtained are displayed in Table 5, along with the average prediction variances of Laake's designs and the I-optimal designs. Clearly, the fact that Laake 
Table 4: I-optimal weights for the points of the $\{q, 3\}$ simplex-centroid design for a secondorder model.

\begin{tabular}{|c|ccc|ccc|ccc|ccc|}
\hline \multirow{2}{*}{$i$} & \multicolumn{3}{|c|}{$q=3$} & \multicolumn{3}{c|}{$q=4$} & \multicolumn{3}{c|}{$q=5$} & \multicolumn{3}{c|}{$q=6$} \\
\cline { 2 - 12 } & $n_{i}$ & $w_{i}$ & $n_{i} w_{i}$ & $n_{i}$ & $w_{i}$ & $n_{i} w_{i}$ & $n_{i}$ & $w_{i}$ & $n_{i} w_{i}$ & $n_{i}$ & $w_{i}$ & $n_{i} w_{i}$ \\
\hline 1 & 3 & 0.1002 & 0.3005 & 4 & 0.0515 & 0.2060 & 5 & 0.0314 & 0.1571 & 6 & 0.0218 & 0.1310 \\
2 & 3 & 0.2016 & 0.6047 & 6 & 0.0947 & 0.5681 & 10 & 0.0493 & 0.4932 & 15 & 0.0277 & 0.4156 \\
3 & 1 & 0.0949 & 0.0949 & 4 & 0.0565 & 0.2258 & 10 & 0.0350 & 0.3497 & 20 & 0.0227 & 0.4534 \\
\hline$d$ & \multicolumn{4}{|c|}{7} & & \multicolumn{4}{|c|}{14} & & \multicolumn{3}{|c|}{25} & & \multicolumn{3}{|c|}{41} \\
\hline
\end{tabular}

Table 5: Average prediction variances of Laake's designs for the second-order model and the continuous I-optimal designs displayed in Table 4, as well as the relative I-efficiencies of Laake's designs.

\begin{tabular}{|l|c|c|c|}
\hline \multirow{2}{*}{$q$} & \multicolumn{2}{|c|}{ Average Prediction Variance } & \multirow{2}{*}{ I-efficiency } \\
\cline { 2 - 4 } & Laake's design & I-optimal design & \\
\hline 3 & 3.2856 & 3.2406 & 0.9863 \\
4 & 4.5550 & 4.3081 & 0.9458 \\
5 & 5.9524 & 5.3290 & 0.8953 \\
6 & 7.3805 & 6.2976 & 0.8533 \\
\hline
\end{tabular}

(1975) did not consider the ternary mixtures has a detrimental effect on the I-efficiency, especially when $q \geq 5$.

The continuous I-optimal design for $q=2$ has weight 0.25 for each of the two pure components and 0.5 for the binary mixture. These weights differ from those produced by Liu and Neudecker (1995), 0.3 and 0.4, respectively. Our I-optimal design for $q=2$ has an average variance of prediction of 2.1333, as opposed to 2.2222 for Liu and Neudecker's design. The I-efficiency of Liu and Neudecker's design relative to the I-optimal design is therefore $96 \%$.

Our continuous I-optimal design for $q=3$ matches the one continuous I-optimal mixture design reported in Wang et al. (2012) for the second-order model in a series of proofof-concept examples for a particle swarm optimization algorithm for finding D-, A- and I-optimal mixture designs. 
The insight that continuous I-optimal designs for the second-order model involve design points other than pure components and binary mixtures is new to the literature. This finding is of importance to researchers who use a point-exchange algorithm for constructing exact I-optimal designs. The output quality of such an algorithm to a large extent depends on the user-specified set of candidate design points. The results in Table 4 imply that the set of candidate points for constructing exact designs should also involve ternary mixtures when the interest is in the second-order model.

\subsection{Special cubic model}

While the continuous D-optimal designs' points for special cubic models are those from the $\{q, 3\}$ simplex-centroid design for $q \geq 3$, this is not true for the continuous I-optimal designs' points when $q \geq 4$. For $q=4$, the full $\{4,4\}$ simplex-centroid design is needed,

and, for $q \geq 5$, the $\{q, 5\}$ simplex-centroid designs' points are needed. The I-optimal weights for the pure components, the binary mixtures, the ternary mixtures and any other required higher-order mixtures are shown in Table 6. For $q$ values ranging from 4 to $6,9.9 \%$ up to $35.3 \%$ of the observations has to be taken at points that are not pure components, binary mixtures or ternary mixtures. The I-efficiencies of the designs proposed by Laake (1975) relative to the I-optimal designs we obtained are displayed in Table 7, along with the average prediction variances for the two sets of designs. Clearly, the fact that Laake (1975) only considered the points of the $\{q, 3\}$ simplex-centroid design has a negative impact on the I-efficiency of the designs he obtained, especially when $q=6$.

Note that, for $q=3$, the weights of our I-optimal continuous design are identical to those obtained by Laake (1975) and to those of Wang et al. (2012), who present exactly one I-optimal design for a special cubic model. Our weights are therefore different from those produced by the analytical expressions in Liu and Neudecker (1995). The design of Liu 
Table 6: I-optimal weights for the points of the $\{q, 5\}$ simplex-centroid design for a special cubic model.

\begin{tabular}{|c|c|c|c|c|c|c|c|c|c|c|c|c|}
\hline \multirow{2}{*}{$i$} & \multicolumn{3}{|c|}{$q=3$} & \multicolumn{3}{|c|}{$q=4$} & \multicolumn{3}{|c|}{$q=5$} & \multicolumn{3}{|c|}{$q=6$} \\
\hline & $n_{i}$ & $w_{i}$ & $n_{i} w_{i}$ & $n_{i}$ & $w_{i}$ & $n_{i} w_{i}$ & $n_{i}$ & $w_{i}$ & $n_{i} w_{i}$ & $n_{i}$ & $w_{i}$ & $n_{i} w_{i}$ \\
\hline 1 & 3 & 0.0925 & 0.2775 & 4 & 0.0426 & 0.1704 & 5 & 0.0227 & 0.1135 & 6 & 0.0134 & 0.0806 \\
\hline 2 & 3 & 0.1483 & 0.4449 & 6 & 0.0557 & 0.3342 & 10 & 0.0248 & 0.2480 & 15 & 0.0125 & 0.1881 \\
\hline 3 & 1 & 0.2776 & 0.2776 & 4 & 0.0991 & 0.3964 & 10 & 0.0409 & 0.4090 & 20 & 0.0189 & 0.3789 \\
\hline 4 & & & & 1 & 0.0990 & 0.0990 & 5 & 0.0413 & 0.2065 & 15 & 0.0185 & 0.2774 \\
\hline 5 & & & & & & & 1 & 0.0233 & 0.0233 & 6 & 0.0125 & 0.0751 \\
\hline$d$ & & 7 & & & 15 & & & 31 & & & 62 & \\
\hline
\end{tabular}

Table 7: Average prediction variances of Laake's designs for the special cubic model and the continuous I-optimal designs displayed in Table 6, as well as the I-efficiencies of Laake's designs relative to the I-optimal ones.

\begin{tabular}{|c|c|c|c|}
\hline \multirow{2}{*}{$q$} & \multicolumn{2}{|c|}{ Average Prediction Variance } & \multirow{2}{*}{ I-efficiency } \\
\cline { 2 - 3 } & Laake's design & I-optimal design & \\
\hline 3 & 3.7543 & 3.7543 & 1.0000 \\
4 & 6.1690 & 5.8607 & 0.9500 \\
5 & 10.0687 & 8.4022 & 0.8345 \\
6 & 15.9957 & 11.3257 & 0.7080 \\
\hline
\end{tabular}

and Neudecker for $q=3$ has a relative I-efficiency of $90.87 \%$ only.

The fact that, for $q \geq 4$, the I-optimal continuous designs contain quaternary and quinary mixtures is new to the literature. It implies that the set of candidate points when constructing exact designs for the special cubic model using a point-exchange algorithm should also involve these types of mixtures.

\section{Exact I-optimal designs}

In this section, we study to what extent exact I-optimal designs differ from continuous ones. More specifically, we investigate whether the design points of the exact designs equal those of the continuous designs. For D-optimal mixture designs, it is known that, for any number of runs $n$, the points of the exact designs are identical to those for the 
continuous designs. We also study the performance of I-optimal exact designs under the D-optimality criterion and of D-optimal exact designs under the I-optimality criterion. The exact designs discussed in this section were all obtained using 1,000,000 runs of the mixture coordinate-exchange algorithm of Piepel et al. (2005), which we adapted to calculate I-optimal instead of D-optimal designs. Unlike point-exchange algorithms, this algorithm does not require specification of a candidate set. This is a major advantage when the location of the points of an optimal design is uncertain.

\subsection{Three ingredients}

This section studies I-optimal designs for the second-order model in case $q=3$. We start by discussing a scenario with many runs, and then consider three scenarios with small numbers of runs.

\subsubsection{0 runs}

The I-optimal 30-run design involves three replicates of the three pure components and the ternary mixture, and six replicates of the three binary mixtures. In this case, the exact I-optimal design thus has seven distinct design points, which match those of the continuous I-optimal design. The D-optimal 30-run design does not involve the ternary mixture and has five replicates of the pure components and the binary mixtures. The D-optimal design therefore does not collect information in the center of the experimental region, unlike the I-optimal design. In this scenario, involving 30 runs, both the I-optimal design and the D-optimal design are symmetric and unique. The D-efficiency of the Ioptimal design relative to the D-optimal design equals $89.02 \%$, whereas the I-efficiency of the D-optimal design relative to the I-optimal design equals $85.79 \%$. The average variances of prediction are 0.1086 and 0.1265 for the I- and D-optimal designs, respectively. 
In addition to showing the location of the design points, Figure 1 compares the prediction variances produced by the two designs over the entire experimental region. White areas correspond to a scaled prediction variance below 2.5. Light gray areas correspond to a scaled prediction variance between 2.5 and 3.5. Dark gray areas correspond to a scaled prediction variance between 4.5 and 5.5. Black areas correspond to a scaled prediction variance above 5.5. From the figure, it is clear that the I-optimal design leads to smaller prediction variances over most of the experimental region. Figure 2 compares the Fraction of Design Space (FDS) plots for the two designs. The figure confirms that the I-optimal design (represented by the solid line) outperforms the D-optimal one (represented by the dashed line) for large portions of the experimental region. The figure also shows that the I-optimal design has a median prediction variance below 0.10 , whereas that of the D-optimal design is about 0.125. The maximum prediction variance is largest for the I-optimal design. The I-optimal design results in large prediction variances in the corners of the experimental region, because it has fewer replicates of the pure components and therefore collects less information in the experimental region's corners.

\subsubsection{6, 7 and 8 runs}

When $n=6$ and the second-order Scheffé model is used, it is impossible for the exact I-optimal design to contain the points of the continuous I-optimal design, simply because the latter design involves seven distinct points. Therefore, the exact I-optimal 6-run design equals the exact D-optimal design, i.e., the $\{3,2\}$ simplex-lattice design in Figure $3 a$. The design has an average prediction variance of 0.6354 .

When $n=7$, the exact I-optimal design is the $\{3,3\}$ simplex-centroid design in Figure $3 \mathrm{~b}$. It thus involves the same design points as the continuous I-optimal design. It has an average variance of prediction is 0.4952 . While there is only one I-optimal design, there 


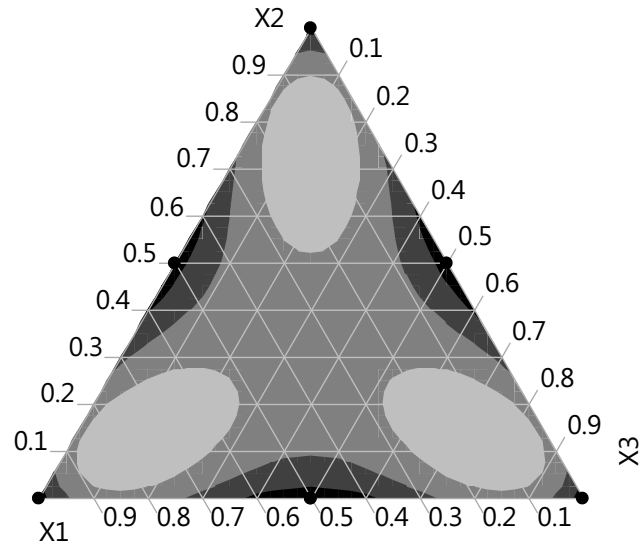

(a) D-optimal design

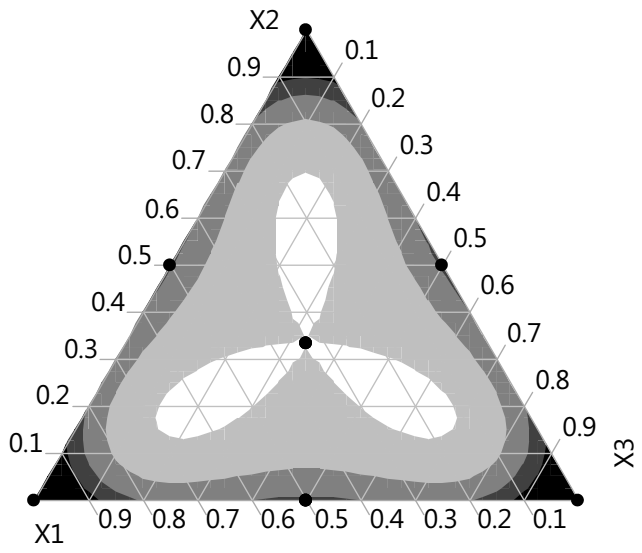

(b) I-optimal design

Figure 1: Comparison of the prediction variances produced by the 30-run 3-ingredient Dand I-optimal designs for a second-order model. White areas correspond to a small scaled prediction variance (below 2.5), while black areas correspond to a large scaled prediction variance (above 5.5). The dots in the figure correspond to the design points. All design points are replicated in the optimal designs, but this not happen equally often in the I-optimal design.

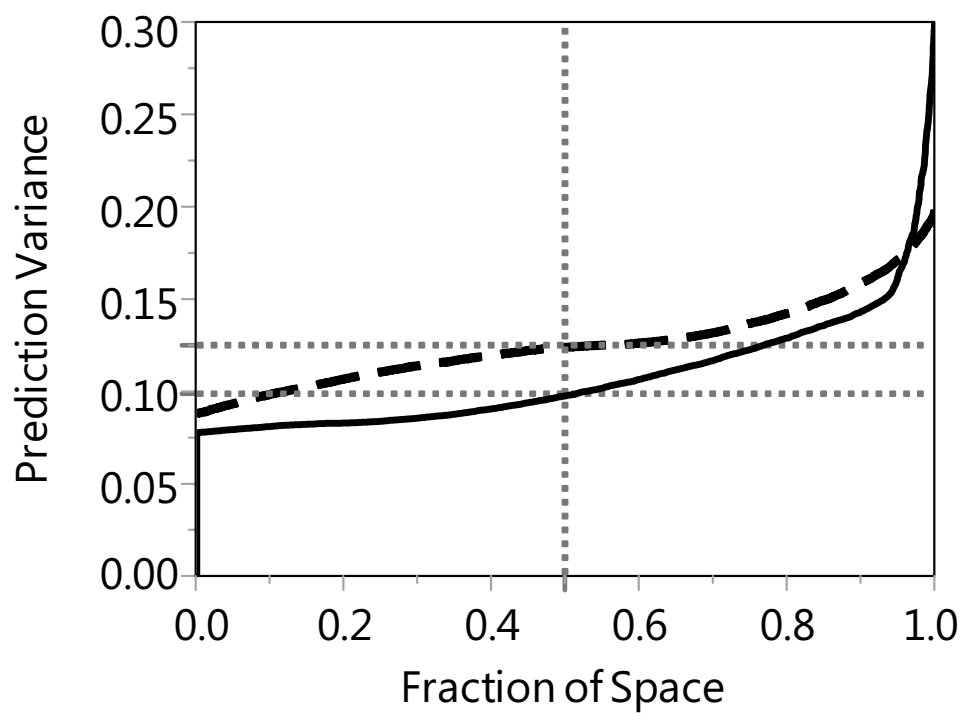

Figure 2: FDS plots for the 30-run 3-ingredient D-optimal design (dashed line) and Ioptimal design (solid line) for a second-order model. 


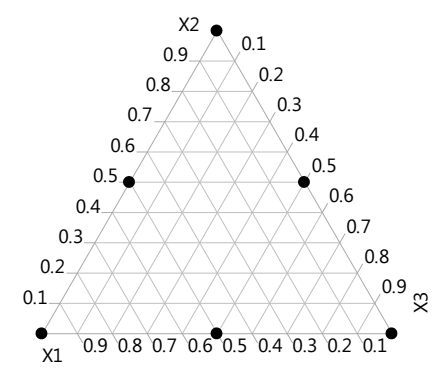

(a) 6 runs

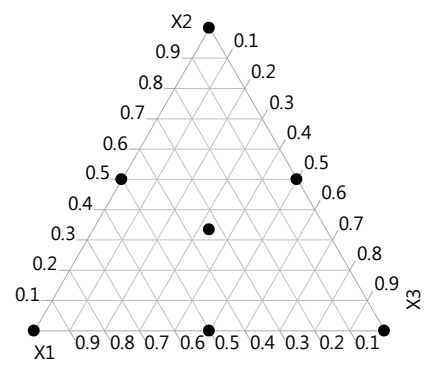

(b) 7 runs

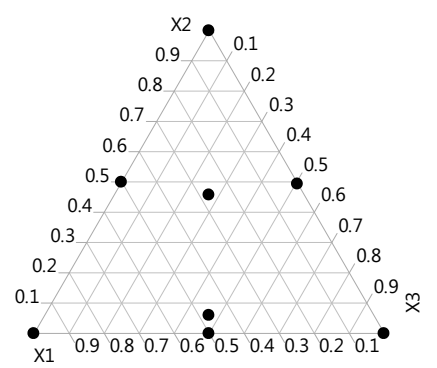

(c) 8 runs

Figure 3: Small I-optimal 3-ingredient designs for a second-order model.

are six different exact D-optimal designs when $n=7$. All of these involve the six points of the $\{3,2\}$ simplex-lattice design, but they differ in the point they replicate and in terms of the I-optimality criterion:

- The three D-optimal designs that replicate a pure component have an average prediction variance of 0.6186 . The I-efficiency of these designs is $80.05 \%$.

- The three D-optimal designs that replicate a binary mixture have an average prediction variance of 0.5468 . The I-efficiency of these designs is $90.57 \%$.

Thus, there is a major difference in I-efficiency between the two types of D-optimal designs. The D-efficiency of the I-optimal design relative to the D-optimal designs is $96.64 \%$.

The performance of the two types of D-optimal designs and the I-optimal design is compared in Figure 4. The dashed line corresponds to the D-optimal designs involving a replicated pure component. The dash-dotted line corresponds to the D-optimal designs with a replicated binary mixture. Finally, the solid line corresponds to the I-optimal design. The dashed horizontal lines in the figure indicate the median prediction variances for the three designs.

When $n=8$, there are three I-optimal designs with an average prediction variance of 


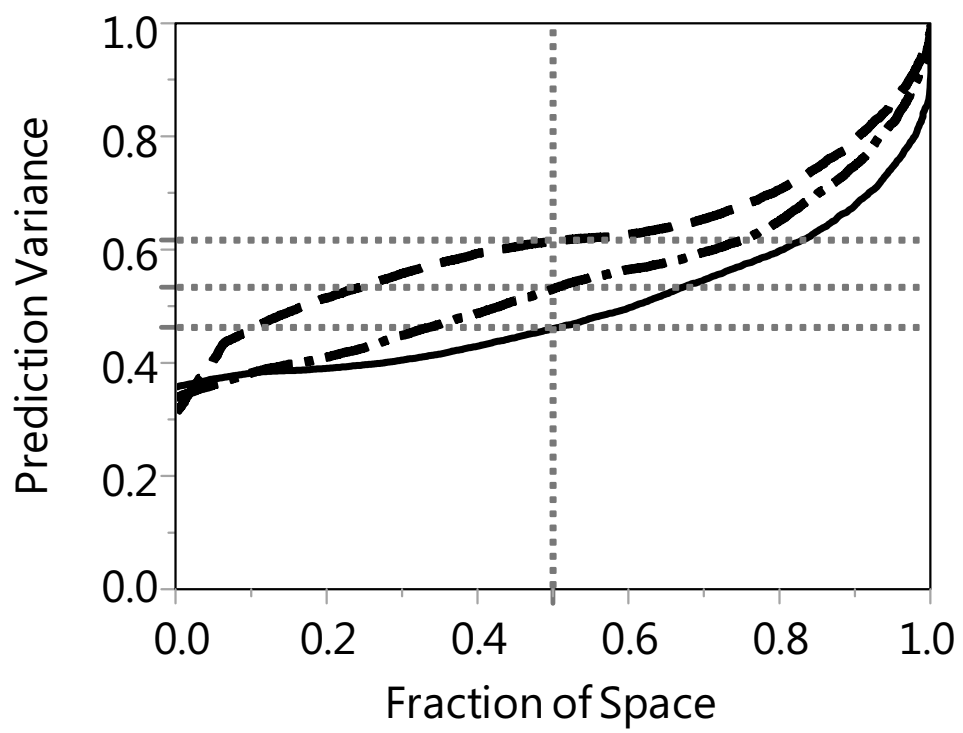

Figure 4: FDS plots for the 7-run 3-ingredient D- and I-optimal designs for a second-order model. The I-optimal design is represented by the solid line. The D-optimal designs that replicate a pure component and those that replicate a binary mixture are represented by the dashed and dash-dotted lines, respectively.

0.4371, one of which is shown in Figure 3c. The other two I-optimal designs can be obtained by cyclically permuting the labels of the ingredients in the figure. Two of the Ioptimal designs' points, located in the interior of the simplex, deviate substantially from those of the simplex-centroid design. In case $n=8$, there are three different types of D-optimal designs:

- One type of D-optimal design replicates two of the three pure components and no binary mixtures. It has an average prediction variance of 0.6000 and a relative I-efficiency of $72.85 \%$.

- A second type of D-optimal design replicates one of the pure components and one of the binary mixtures. It has an average prediction variance of 0.5278 and a relative I-efficiency of $82.82 \%$.

- A third type of D-optimal design replicates two of the three binary mixtures and 
no pure components. It has an average prediction variance of 0.4556 and a relative I-efficiency of $95.94 \%$.

The D-optimal designs' performance in terms of I-efficiency thus increases with the number of replicated binary mixtures. The I-optimal designs for $n=8$ have a D-efficiency of $94.08 \%$ relative to the D-optimal designs.

The performance of the three types of D-optimal designs in terms of prediction variance is compared graphically to that of the I-optimal designs in Figure 5. The long-dashed, dashdotted and short-dashed lines correspond to the D-optimal designs with two replicated pure components, one replicated pure component and no replicated pure components, respectively. The solid black line corresponds to the three I-optimal designs. The plot shows that there is a large difference in performance in terms of prediction variance between the three types of D-optimal designs. The dashed horizontal lines indicate that the D-optimal designs without replicated pure components almost have the same median prediction variance as the I-optimal designs. The D-optimal designs with two replicated pure components, however, have a median variance of prediction that is about $50 \%$ larger than that of the I-optimal designs.

\subsection{Four ingredients}

\subsubsection{A 15-run design for the second-order model}

The patterns we observed for three ingredients and a second-order model persist in case the number of ingredients is increased. We illustrate this with a 15-run example involving four ingredients. One I-optimal design for this scenario is shown in Table 8. It yields an average prediction variance of 0.3022 . All D-optimal designs for this scenario involve the four pure components and the six binary mixtures, and replicate five of these ten 


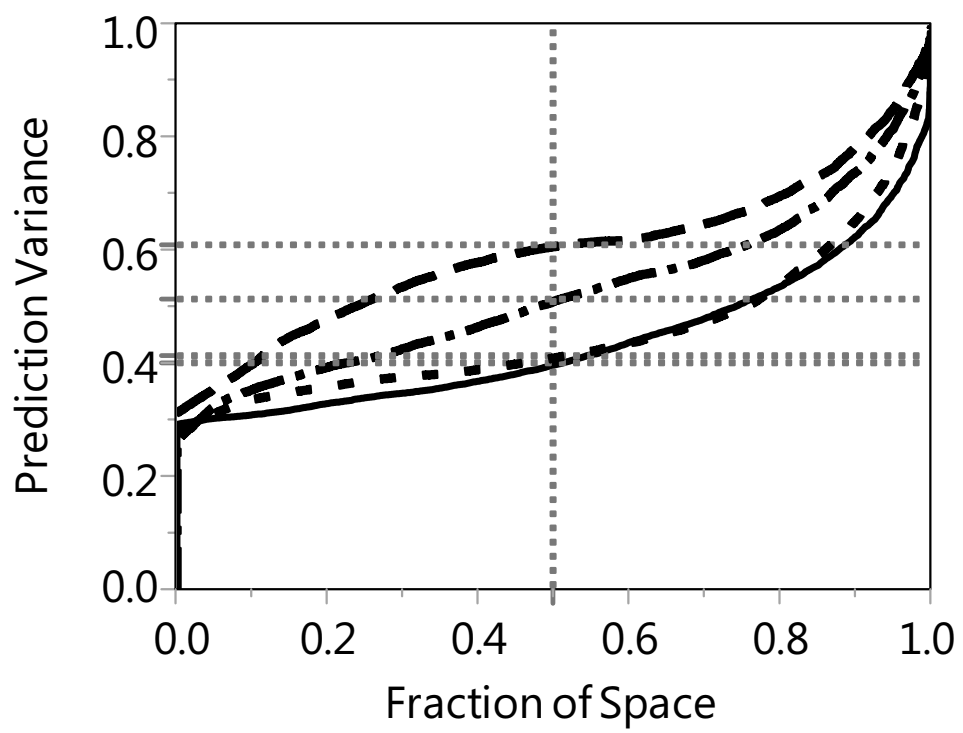

Figure 5: FDS plots for the 8-run 3-ingredient D- and I-optimal designs for a second-order Scheffé model. The long-dashed, dash-dotted and short-dashed lines correspond to the Doptimal designs with two replicated pure components, one replicated pure component and no replicated pure components, respectively. The solid line corresponds to the I-optimal designs.

design points. There are five types of D-optimal designs, which can be characterized by the number of pure components they replicate. At one end of the spectrum, there is one type of D-optimal design which replicates each of the four pure components, and one of the binary mixtures. Of all D-optimal designs, this type has the largest possible number of pure component replicates and the smallest possible number of replicates of binary mixtures. At the other end of the spectrum, some D-optimal designs replicate none of the pure components and five of the six binary mixtures. We compare the performance of the five types of D-optimal designs in Table 9. In that table, the five types of D-optimal designs appear in decreasing order of the number of pure component replicates.

The table shows that the different types of D-optimal designs perform quite differently in terms of the I-optimality criterion. The I-efficiency of the D-optimal designs with four pure component replicates and one replicated binary mixture, for instance, amounts to 
Table 8: 15-run I-optimal 4-ingredient design for a second-order model.

\begin{tabular}{|c|cccc|}
\hline Run & $x_{1}$ & $x_{2}$ & $x_{3}$ & $x_{4}$ \\
\hline 1 & 1.0000 & 0.0000 & 0.0000 & 0.0000 \\
2 & 0.5000 & 0.5000 & 0.0000 & 0.0000 \\
3 & 0.5000 & 0.5000 & 0.0000 & 0.0000 \\
4 & 0.0000 & 1.0000 & 0.0000 & 0.0000 \\
5 & 0.2876 & 0.2876 & 0.4248 & 0.0000 \\
6 & 0.5050 & 0.0000 & 0.4950 & 0.0000 \\
7 & 0.0000 & 0.5000 & 0.5000 & 0.0000 \\
8 & 0.0000 & 0.0000 & 1.0000 & 0.0000 \\
9 & 0.3331 & 0.0000 & 0.3335 & 0.3335 \\
10 & 0.0000 & 0.3326 & 0.3337 & 0.3337 \\
11 & 0.2885 & 0.2885 & 0.0000 & 0.4230 \\
12 & 0.0000 & 0.5066 & 0.0000 & 0.4934 \\
13 & 0.5000 & 0.0000 & 0.0000 & 0.5000 \\
14 & 0.0000 & 0.0000 & 0.5000 & 0.5000 \\
15 & 0.0000 & 0.0000 & 0.0000 & 1.0000 \\
\hline
\end{tabular}

Table 9: Detailed comparison of the I-optimal design and the five types of D-optimal designs involving 15 runs for the 4-ingredient second-order model.

\begin{tabular}{|l|c|c|cc|c|}
\hline \multirow{2}{*}{ Design } & Replicated & Replicated & Prediction Variance & \multirow{2}{*}{ I-efficiency } \\
\cline { 4 - 5 } & Pure Comp. & Bin. Mixt. & Average & Median & \\
\hline D-optimal 1 & 4 & 1 & 0.4476 & 0.4269 & $67.33 \%$ \\
D-optimal 2 & 3 & 2 & 0.4167 & 0.3886 & $72.33 \%$ \\
D-optimal 3 & 2 & 3 & 0.3857 & 0.3586 & $78.13 \%$ \\
D-optimal 4 & 1 & 4 & 0.3548 & 0.3287 & $84.95 \%$ \\
D-optimal 5 & 0 & 5 & 0.3238 & 0.3015 & $93.08 \%$ \\
\hline I-optimal & & & 0.3014 & 0.2821 & $100.00 \%$ \\
\hline
\end{tabular}


$67.33 \%$ only, while that of the D-optimal designs without pure component replicates (but with five replicated binary mixtures) is $93.08 \%$. The I-efficiency of the D-optimal design thus increases substantially with the number of replicates of binary mixtures. The Defficiency of the I-optimal design equals $90.51 \%$.

An important feature of the I-optimal design in Table 8 is that it involves points different from those of the $\{q, 2\}$ simplex-lattice and $\{q, 3\}$ simplex-centroid designs. These points are of a kind typically not included in candidate sets for point-exchange algorithms, so that it is virtually impossible to obtain this design by means of a point-exchange algorithm.

\subsubsection{6- and 17-run designs for the special cubic model}

We also computed exact I-optimal 4-ingredient designs for the special cubic model. In some cases, the exact I-optimal designs' points match those of the continuous I-optimal designs, while, in other cases, the exact designs involve a few points that do not occur in the continuous I-optimal design.

For $n=16$, for instance, the exact I-optimal 4-ingredient design we obtained involves one run with each pure component, each binary mixture and each ternary mixture, and two runs with the quaternary mixture. Hence, for $n=16$, the points of the exact I-optimal design match those of the continuous I-optimal design. There are six types of 16-run Doptimal designs for the special cubic model, all of which involve the four pure components, the six binary mixtures and the four ternary mixtures, but not the quaternary mixture. At one end of the spectrum, one type of D-optimal design involves two replicates of two different pure components. At the other end of the spectrum, another type involves two replicates of two ternary mixtures. All types of D-optimal designs perform differently in terms of the I-optimality criterion, the best type being that with two replicated ternary 
Table 10: Detailed comparison of the I-optimal design and the six types of D-optimal designs involving 16 runs for the 4-ingredient special cubic model.

\begin{tabular}{|l|c|c|c|cc|c|}
\hline \multirow{2}{*}{ Design } & \multicolumn{3}{|c|}{ Replicates } & \multicolumn{2}{c|}{ Prediction Variance } & \multirow{2}{*}{ I-efficiency } \\
\cline { 2 - 6 } & Pure Comp. & Bin. Mixt. & Tern. Mixt. & Average & Median & \\
\hline D-optimal 1 & 2 & 0 & 0 & 0.5321 & 0.5215 & $75.01 \%$ \\
D-optimal 2 & 1 & 1 & 0 & 0.5280 & 0.5151 & $75.60 \%$ \\
D-optimal 3 & 1 & 0 & 1 & 0.4893 & 0.4676 & $81.58 \%$ \\
D-optimal 4 & 0 & 2 & 0 & 0.5238 & 0.5098 & $76.20 \%$ \\
D-optimal 5 & 0 & 1 & 1 & 0.4893 & 0.4601 & $81.58 \%$ \\
D-optimal 6 & 0 & 0 & 2 & 0.4464 & 0.4206 & $89.41 \%$ \\
\hline I-optimal & & & & 0.3992 & 0.3684 & $100.00 \%$ \\
\hline
\end{tabular}

mixtures. That design has an I-efficiency of $89.41 \%$ relative to the I-optimal design. The performance of the D-optimal designs in terms of the I-optimality criterion especially increases with the number of replicates of the ternary mixtures. This is shown in detail in Table 10, where the different types of D-optimal designs appear in decreasing order of the number of replicated pure components, and, in case that number is the same, in decreasing order of the number of replicated binary mixtures.

For $n=17$, the exact I-optimal 4-ingredient design we obtained involves one run with each pure component and each binary mixture, essentially two runs with one of the ternary mixtures and one run with each of the remaining ternary mixtures. Additionally, there are two runs involving nonzero, unequal proportions for each of the four ingredients. That $17-$ run I-optimal design is displayed in Table 11. This design shows that it is not sufficient to consider the points of the continuous I-optimal design for constructing an exact I-optimal design for a special cubic model.

\section{Discussion}

In this article, we provide the first thorough literature review concerning the I-optimal design of mixture experiments. It turns out that the continuous designs previously proposed 
Table 11: 17-run I-optimal 4-ingredient design for a special cubic model.

\begin{tabular}{|c|cccc|}
\hline Run & $x_{1}$ & $x_{2}$ & $x_{3}$ & $x_{4}$ \\
\hline 1 & 1.0000 & 0.0000 & 0.0000 & 0.0000 \\
2 & 0.5000 & 0.5000 & 0.0000 & 0.0000 \\
3 & 0.0000 & 1.0000 & 0.0000 & 0.0000 \\
4 & 0.3358 & 0.3284 & 0.3358 & 0.0000 \\
5 & 0.5000 & 0.0000 & 0.5000 & 0.0000 \\
6 & 0.0000 & 0.5000 & 0.5000 & 0.0000 \\
7 & 0.0000 & 0.0000 & 1.0000 & 0.0000 \\
8 & 0.2897 & 0.3021 & 0.2041 & 0.2041 \\
9 & 0.1653 & 0.3034 & 0.2656 & 0.2656 \\
10 & 0.3338 & 0.0000 & 0.3331 & 0.3331 \\
11 & 0.3334 & 0.0000 & 0.3333 & 0.3333 \\
12 & 0.3351 & 0.3299 & 0.0000 & 0.3351 \\
13 & 0.0000 & 0.3298 & 0.3351 & 0.3351 \\
14 & 0.0000 & 0.5000 & 0.0000 & 0.5000 \\
15 & 0.0000 & 0.0000 & 0.5000 & 0.5000 \\
16 & 0.5000 & 0.0000 & 0.0000 & 0.5000 \\
17 & 0.0000 & 0.0000 & 0.0000 & 1.0000 \\
\hline
\end{tabular}

in the literature are suboptimal, either because they are based on too few distinct design points (Laake, 1975) or on the incorrect design points (Lambrakis, 1968a) or because the weights of the design points are incorrect (Liu and Neudecker, 1995). We used the multiplicative algorithm to find verifiably I-optimal continuous designs for second-order and special cubic Scheffé models, and the general equivalence theorem to confirm the I-optimality.

An important result of our work is that the points of the $\{q, 3\}$ simplex-centroid design are relevant if the goal of the experimenter is to minimize the average variance of prediction for a second-order model. This insight is surprising, because, following the work of Scheffé (1958) and a known result concerning D-optimality (Kiefer, 1961), experimenters associate the $\{q, 2\}$ simplex-lattice design rather than the $\{q, 3\}$ simplex-centroid design with the second-order model. Another important result of our work is that the points 
of the $\{q, 5\}$ simplex-centroid design are relevant if the goal is to minimize the average variance of prediction for a special cubic model. This insight is also surprising, because, following the work of Scheffé (1958) and another known result concerning D-optimality (Uranisi, 1964), experimenters associate the $\{q, 3\}$ simplex-centroid design rather than the $\{4,4\}$ and $\{q, 5\}$ simplex-centroid designs with the special cubic model in case $q=4$ and $q \geq 5$, respectively.

Our computational results for exact I-optimal designs show that the use of point-exchange algorithms is bound to lead to suboptimal designs, even in case the design region is simplex-shaped. This was demonstrated by the I-optimal designs in Figure 2 and Tables 8 and 11. For the construction of I-optimal designs for mixture experiments, we strongly recommend using an algorithm that is not based on a set of candidate design points, such as the mixture coordinate-exchange algorithm of Piepel et al. (2005).

In the course of the research that led to this paper, we also employed the SAS procedure OPTEX for generating exact I-optimal mixture designs, inspired by the online documentation which states that the I-optimality criterion is equivalent to the A-optimality criterion when the candidate set is orthogonally coded. In our view, the use of the SAS procedure OPTEX has two weaknesses when it comes to generating I-optimal designs. First, it is based on a point-exchange algorithm. It will therefore produce suboptimal designs in many situations. Second, even assuming the candidate set contains all the points required to construct an I-optimal design, the SAS procedure OPTEX will fail to do so in most practical instances, because it does not take into account the correct moments matrix B. Instead, the OPTEX procedure implicitly works with an approximate moments matrix based on the candidate set specified. A simple example of a situation in which opTEX fails is one where a candidate set involving the points of the $\{3,3\}$ simplex-centroid 
design is specified and a 1,000-run I-optimal design for a second-order model is desired. In that case, SAS produces designs that do not include the ternary mixture, even though the continuous I-optimal design has a weight of $9.5 \%$ for the ternary mixture (see Table 4 ) and the 1,000-run I-optimal design should, therefore, involve 95 replicates of the ternary mixture. Using a larger candidate set, for instance by including the ternary mixture several times in the candidate set, leads to a different design, including the ternary point. For instance, including twelve copies of the ternary mixture in the candidate set leads SAS to produce a 1,000-run design with 222 replicates of the ternary mixture, more than twice as many as is I-optimal. This shows that the OPTEX procedure is unreliable when it comes to generating I-optimal designs.

We focused on I-optimal designs for second-order and special cubic Scheffé models in this article, because these are most commonly models for data from mixture experiments. Scheffé also suggested the less commonly used full cubic and $q$-th degree models. For the full cubic model, the designs displayed in Table 3, based on the $\{q, 3\}$ simplex-lattice designs and proposed by Laake (1975), are the best known designs in terms of I-optimality. These designs, however, do not satisfy the general equivalence theorem. As a result, the points of the $\{q, 3\}$ simplex-lattice design are not I-optimal. To look for continuous Ioptimal designs for the full cubic models and to study their relationship to exact designs would be an interesting avenue for future research. For the $q$ th degree model, Liu and Neudecker (1995) analytically derive I-optimal weights for the points of the full simplexcentroid design and claim the resulting designs satisfy the general equivalence theorem. As shown in Section 5.3 for the second-order model and in Section 5.4 for the special cubic model, the designs of Liu and Neudecker for two and three ingredients are not I-optimal. Using the multiplicative algorithm, we have obtained I-optimal designs for the $q$ th degree model for two up to six ingredients. Our I-optimal designs, which are contrasted with Liu 
and Neudecker's designs in the online supplemental file, are based on the full simplexcentroid design and satisfy the general equivalence theorem.

In this paper, we adopted a computational approach to identifying continuous I-optimal designs, and to verify that the resulting designs satisfy the general equivalence theorem. Another interesting topic for future research would be to derive general analytical expressions for the weights in the continuous I-optimal designs, and to prove their optimality analytically.

While our focus was on I-optimal designs, we also provide interesting results concerning D-optimal mixture designs. If an experimenter is interested in D-optimal mixture designs, then there are several options which perform very differently in terms of I-optimality. A similar point was made for D-optimal screening designs by Robinson and Anderson-Cook (2010). It is generally useful to evaluate and optimize experimental designs with respect to more than one optimality criterion. In recent years, this theme has received substantial attention, for example by Lu, Anderson-Cook and Robinson (2011) and Sambo, Borrotti and Mylona (2014).

\section{Acknowledgement}

The authors wish to acknowledge the financial support of the Fonds voor Wetenschappelijk Onderzoek - Vlaanderen. The third author was financially supported by the General Directorat of Higher Education of the Indonesian Ministry of National Education. 


\section{Appendix: The multiplicative algorithm}

Finding a continuous I-optimal design requires finding the design that minimizes $\operatorname{tr}\left(\mathbf{M}^{-1} \mathbf{B}\right)$, where $\mathbf{B}$ is the moments matrix and $\mathbf{M}=\mathbf{X}^{\prime} \mathbf{W X}$ is the continuous designs' information matrix. In this expression, $\mathbf{W}=\operatorname{diag}\left(w_{1}, w_{2}, \ldots, w_{d}\right)$, with $d$ the number of distinct design points. Every weight $w_{i}$ indicates the proportion of runs to be performed at the $i$ th design point (appearing in the $i$ th row of $\mathbf{X}$ ).

The multiplicative algorithm starts from a random set of weights $w_{1}, w_{2}, \ldots, w_{d}$ for a set of $d$ given distinct design points, and improves the weights iteratively. We denote the starting weights by $w_{1}^{0}, w_{2}^{0}, \ldots, w_{d}^{0}$ and the corresponding information matrix by $\mathbf{M}^{0}$. We denote the weight obtained at the $r$ th iteration by $w_{1}^{r}, w_{2}^{r}, \ldots, w_{d}^{r}$ and the corresponding information matrix by $\mathbf{M}^{r}$.

The iterative improvement of the weights is guided by the general equivalence theorem in (10), which states that $\mathbf{f}^{\prime}(\mathbf{x}) \mathbf{M}^{-1} \mathbf{B} \mathbf{M}^{-1} \mathbf{f}(\mathbf{x})=\operatorname{tr}\left(\mathbf{M}^{-1} \mathbf{B}\right)$ for each design point:

- The weight of a design point $\mathbf{x}$, for which $\mathbf{f}^{\prime}(\mathbf{x}) \mathbf{M}^{-1} \mathbf{B} \mathbf{M}^{-1} \mathbf{f}(\mathbf{x})>\operatorname{tr}\left(\mathbf{M}^{-1} \mathbf{B}\right)$, is too low and should be increased.

- The weight of a design point $\mathbf{x}$, for which $\mathbf{f}^{\prime}(\mathbf{x}) \mathbf{M}^{-1} \mathbf{B} \mathbf{M}^{-1} \mathbf{f}(\mathbf{x})<\operatorname{tr}\left(\mathbf{M}^{-1} \mathbf{B}\right)$, is too high and should be decreased.

The outline of the multiplicative algorithm for I-optimality is as follows:

1. Randomly generate $w_{1}^{0}, w_{2}^{0}, \ldots, w_{d}^{0}$ and set $r=0$.

2. Repeat until the maximum change in weight is smaller than a prespecified value $\epsilon$ :

(a) Set $r=r+1$. 
(b) For each of the $d$ distinct design points $\mathbf{x}_{i}$, update the weight using the following formula:

$$
w_{i}^{r+1}=w_{i}^{r}\left(\frac{\mathbf{f}^{\prime}\left(\mathbf{x}_{i}\right)\left(\mathbf{M}^{r}\right)^{-1} \mathbf{B}\left(\mathbf{M}^{r}\right)^{-1} \mathbf{f}\left(\mathbf{x}_{i}\right)}{\operatorname{tr}\left(\left(\mathbf{M}^{r}\right)^{-1} \mathbf{B}\right)}\right)^{\delta}
$$

where $\delta$ is a convergence parameter.

Following Fellman (1974), for our computations, we set $\delta$ to 0.5. If this algorithm converges to a set of weights which ensure that the equality in (10) holds, then an I-optimal design is found. If the algorithm does not return such a set of weights, it means that a different set of distinct design points is required to find the I-optimal design.

\section{References}

Atkinson, A. C., Donev, A. N. and Tobias, R. D. (2007). Optimum Experimental Designs, with $S A S$, Oxford: Oxford University Press.

Atwood, C. L. (1969). Optimal and efficient designs of experiments, The Annals of Mathematical Statistics 40: 1570-1602.

Becker, N. G. (1968). Models for the response of a mixture, Journal of the Royal Statistical Society, Series B 30: 349-358.

Borkowski, J. J. (2003). Using a genetic algorithm to generate small exact response surface designs, Journal of Probablity and Statistical Science 1: 65-88.

Chan, L.-Y. (1995). A review on optimal design for mixture model, in K. Chan and M. Liu (eds), Five Decades As A Mathematician And Educator: On the 80th Birthday of Professor Yung-Chow Wong, World Scientific Singapore.

Cornell, J. A. (2002). Experiments with Mixtures: Designs, Models, and the Analysis of Mixture Data, New York: Wiley. 
DeGroot, M. (1970). Optimal Statistical Decisions, New York: McGraw Hill.

Fellman, J. (1974). On the allocation of linear observations, Commentationes PhysicoMathematicae 44: 27-78.

Giovannitti-Jensen, A. and Myers, R. H. (1989). Graphical assessment of the prediction capability of response surface designs, Technometrics 31: 159-171.

Goldfarb, H. B., Anderson-Cook, C. M., Borror, C. M. and Montgomery, D. C. (2004). Fraction of design space plots for assessing mixture and mixture-process designs, Journal of Quality Technology 36: 169-179.

Goos, P. and Jones, B. (2011). Design of Experiments: A Case Study Approach, Wiley.

Haines, L. M. (1987). The application of the annealing algorithm to the construction of exact optimal designs for linear-regression models, Technometrics 29: 439-447.

Hardin, R. H. and Sloane, N. J. A. (1993). A new approach to construction of optimal designs, Journal of Statistical Planning and Inference 37: 339-369.

Kiefer, J. (1961). Optimum design in regression problems II, Annals of Mathematical Statistics 32: 298-325.

Kiefer, J. and Wolfowitz, J. (1960). The equivalence of two extremum problems, Canadian Journal of Mathematics 12: 363-366.

Laake, P. (1975). On the optimal allocation of observation in experiments with mixtures, Scandinavian Journal of Statistics 2: 153-157.

Lambrakis, D. (1968a). Experiments with mixtures: A generalization of the simplex-lattice design, Journal of Royal Statistical Society. Series B 30(1): 123-136.

Lambrakis, D. (1968b). Experiments with p-component mixtures, Journal of Royal Statistical Society. Series B 30(1): 137-144. 
Letsinger, J. D., Myers, R. H. and Lentner, M. (1996). Response surface methods for birandomization structures, Journal of Quality Technology 28: 381-397.

Liu, S. and Neudecker, H. (1995). A V-optimal design for Scheffé's polynomial model, Statistics and Probability Letters 23: 253-258.

Liu, S. and Neudecker, H. (1997). Experiments with mixtures : Optimal allocations for Becker's models, Metrika 45: 53-66.

Lu, L., Anderson-Cook, C. M. and Robinson, T. J. (2011). Optimization of designed experiments based on multiple criteria utilizing a Pareto frontier, Technometrics 53: 353-365.

Meyer, R. K. and Nachtsheim, C. J. (1988). Simulated annealing in the construction of exact optimal design of experiments, American Journal of Mathematical and Management Science 8: 329-359.

Meyer, R. K. and Nachtsheim, C. J. (1995). The coordinate-exchange algorithm for constructing exact optimal experimental designs, Technometrics 37: 60-69.

Mikaeili, F. (1993). D-optimum design for full cubic on q-simplex, Journal of Statistical Planning and Inference 35: 121-130.

Montgomery, D. C. (2009). Design and Analysis of Experiments, New York: Wiley.

Piepel, G., Cooley, S. and Jones, B. (2005). Construction of a 21-component layered mixture experiment design using a new mixture coordinate-exchange algorithm, Quality Engineering 17: $579-594$.

Robinson, T. J. and Anderson-Cook, C. M. (2010). A closer look at D-optimality for screening designs, Quality Engineering 23: 1-14.

Sambo, F., Borrotti, M. and Mylona, K. (2014). A coordinate-exchange two-phase local search algorithm for the D- and I-optimal designs of split-plot experiments, Computational Statistics E Data Analysis 71: 1193-1207. 
Scheffé, H. (1958). Experiments with mixtures, Journal of the Royal Statistical Society, Ser. B 20: $344-360$.

Scheffé, H. (1963). The simplex-centroid design for experiments with mixtures, Journal of the Royal Statistical Society, Ser. B 25: 235-263.

Silvey, S. D. (1980). Optimal Design, London: Chapman and Hall.

Sinha, B. K., Mandal, N. K., Pal, M. and Das, P. (2014). Optimal Mixture Experiments, New Delhi: Springer.

Smith, W. (2005). Experimental Design for Formulation, Philadelphia: Siam.

Titterington, D. (1976). Algorithms for computing D-optimal design on finite space, Conference on information Science and Systems, Vol. 3, John Hopkins University, pp. 213-216.

Titterington, D. (1978). Estimation of correlation coefficients by ellipsoidal trimming, Applied Statistics 27: 227-234.

Uranisi, H. (1964). Optimal design for the special cubic regression model on the $q$-simplex, Mathematical Report 1, Kyushu University, General Education Department.

Wang, W., Chen, R.-B., Huang, C.-C. and Wong, W.-K. (2012). Particle swarm optimization techniques for finding optimal mixture designs, Preprint, Department of Mathematics, National Taiwan University.

Wynn, H. P. (1972). Results in the theory and construction of D-optimum experimental designs, Journal of the Royal Statistical Society, Ser. B 34: 133-147.

Zahran, A. R., Anderson-Cook, C. M. and Myers, R. H. (2003). Fraction of design space to assess prediction capability of response surface designs, Journal of Quality Technology 35: 377-386. 